\title{
LINEAR RESPONSE OF THE LYAPUNOV EXPONENT TO A SMALL CONSTANT PERTURBATION
}

\author{
RAFAIL V. ABRAMOV
}

\begin{abstract}
AвstRAct. In the current work we demonstrate the principal possibility of prediction of the response of the largest Lyapunov exponent of a chaotic dynamical system to a small constant forcing perturbation via a linearized relation, which is computed entirely from the unperturbed dynamics. We derive the formal representation of the corresponding linear response operator, which involves the (computationally infeasible) infinite time limit. We then compute suitable finite-time approximations of the corresponding linear response operator, and compare its response predictions with actual, directly perturbed and measured, responses of the largest Lyapunov exponent. The test dynamical system is a 20-variable Lorenz 96 model, run in weakly, moderately, and strongly chaotic regimes. We observe that the linearized response prediction is a good approximation for the moderately and strongly chaotic regimes, and less so in the weakly chaotic regime due to intrinsic nonlinearity in the response of the Lyapunov exponent, which the linearized approximation is incapable of following.
\end{abstract}

\section{INTRODUCTION}

The largest Lyapunov exponent (henceforth the Lyapunov exponent) is the cornerstone measure of chaos and uncertainty in complex nonlinear dynamics [19. 29, 30, 36]. It shows the average exponential rate of divergence (if positive) or convergence (if negative) of two nearby solutions of a dynamical system, usually a system of nonlinear differential equations of first order, sometimes with stochastic forcing. Practical methods of computing the Lyapunov exponents from the long-term time series of a dynamical system have been developed in [21, 38].

In this work we develop a linear approximation to the response of the Lyapunov exponent to a small constant perturbation of the corresponding dynamical system. This linear approximation is computed from the long-term time series of the unperturbed dynamics, and, in a way, is a generalization of the well-known Fluctuation-Dissipation theorem [28,31,37]. The advantage of the approach is that it effectively computes all possible responses to all possible perturbations in the corresponding phase space at once, as well as provides a convenient framework for the inverse problem, where the perturbation has to be computed to satisfy the

Date: July 24, 2018.

2000 Mathematics Subject Classification. 37M, 37N. 
given response. The approach we develop here is not generally restricted to constant perturbations, and can be adapted to arbitrary differentiable perturbations of vector fields, provided that the Lyapunov exponent varies sufficiently smoothly under the perturbations.

We consider a system of autonomous nonlinear ordinary differential equations of the form

$$
\frac{\mathrm{d} x}{\mathrm{~d} t}=f(x),
$$

where $x=x(t)$ is a $\mathbb{R}^{N}$-vector valued bounded function of time $t$ for some positive integer $N$, representing the unknown time-dependent solution of the system, and $f(x)$ is a nonlinear differentiable vector field, $f: \mathbb{R}^{N} \rightarrow \mathbb{R}^{N}$.

The largest Lyapunov exponent describes the average exponential rate of separation of solutions $x(t)$ and $y(t)$ of (1.1) for two nearby initial conditions $x_{0}$ and $y_{0}$, in the infinite time limit:

$$
\lambda=\lim _{t \rightarrow \infty} \lim _{y_{0} \rightarrow x_{0}} \frac{1}{t} \ln \frac{\|\boldsymbol{y}(t)-x(t)\|}{\left\|y_{0}-x_{0}\right\|} .
$$

Above, $\|x\|$ denotes the usual Euclidean norm of $x$. The famous Oseledec's multiplicative ergodic theorem [29] states that for almost all, in the appropriate probabilistic sense, starting conditions $x_{0}$, the limit in (1.2) converges to the same value independently of choice of the initial condition $x_{0}$. The relation in (1.2) means that, on average, the distance between two nearby solutions $\boldsymbol{x}(t)$ and $\boldsymbol{y}(t)$ can be estimated as

$$
\|\boldsymbol{y}(t)-\boldsymbol{x}(t)\| \sim e^{\lambda t}\left\|\boldsymbol{y}_{0}-\boldsymbol{x}_{0}\right\| .
$$

Thus, if $\lambda$ is positive, almost any two nearby solutions of (1.1) diverge from each other exponentially rapidly in time, with $\lambda$ being the average exponential rate of divergence. In this case, the dynamical system in (1.1) is said to be chaotic [30,36]. Chaotic dynamical systems can be encountered in fluid dynamics [16], turbulence [22,33] and geophysical science [20,24], and are the subject of our current study.

There is more simple formula for the Lyapunov exponent which excludes the spatial limit. Observe that, for two nearby solutions $\boldsymbol{x}(t)$ and $\boldsymbol{y}(t)$, the difference between them can be approximated as

$$
\begin{gathered}
\frac{\mathrm{d} \boldsymbol{v}}{\mathrm{d} t}=D f(\boldsymbol{x}) \boldsymbol{v}+o(\|\boldsymbol{v}\|), \\
\boldsymbol{v}(t)=\boldsymbol{y}(t)-\boldsymbol{x}(t),
\end{gathered}
$$

where $D f$ is the Jacobian of $f$ (the matrix of partial derivatives of $f$ ). As $\|\boldsymbol{v}\| \rightarrow 0$, the term $o(\|\boldsymbol{v}\|)$ above becomes negligible in comparison with the rest of the 
terms, which results in the linear equation for $v(t)$ in the limit, with $x(t)$ computed in parallel from (1.1):

$$
\frac{\mathrm{d} v}{\mathrm{~d} t}=D f(x) \boldsymbol{v}, \quad \lambda=\lim _{t \rightarrow \infty} \frac{1}{t} \ln \frac{\|\boldsymbol{v}(t)\|}{\left\|v_{0}\right\|} .
$$

Here, $\boldsymbol{v}(t)$ is a tangent vector, with $\left\|\boldsymbol{v}_{0}\right\|=1$, by convention. Here, $\boldsymbol{v}(t)$ does not have to be small, since it can be scaled by an arbitrary constant factor due to the linearity and cancellation in (1.5). For chaotic systems, $\|v(t)\|$ grows exponentially fast, so we avoid the resulting numerical instability with periodic renormalization of $v(t)$ and the corresponding adjustment to $\lambda$ for compensation (for details, see, for example, [19]).

One can also replace the infinite time limit in (1.5) with a time average (or ensemble average, via Birkhoff's theorem [18]). First, factor the tangent vector $v$ from (1.5) into the product of its norm $\|\boldsymbol{v}\|$, and unit vector $w=v /\|v\|$ :

$$
v=\|v\| w .
$$

Then, for the unperturbed system in (1.1), the equation for $\|\boldsymbol{v}(t)\|$ is easily derived as

$$
\frac{\mathrm{d} \ln \|\boldsymbol{v}\|}{\mathrm{d} t}=\boldsymbol{w}^{T} D \boldsymbol{f}(\boldsymbol{x}) \boldsymbol{w} .
$$

The substitution of (1.6) and (1.7) into the formula for the Lyapunov exponent in (1.5) yields, with $n_{0}=1$,

$$
\begin{array}{r}
\lambda=\lim _{t \rightarrow \infty} \frac{1}{t} \ln \|\boldsymbol{v}(t)\|=\lim _{t \rightarrow \infty} \frac{1}{t} \int_{0}^{t} \frac{\mathrm{d} \ln \|\boldsymbol{v}(s)\|}{\mathrm{d} s} \mathrm{~d} s= \\
=\lim _{t \rightarrow \infty} \frac{1}{t} \int_{0}^{t} \boldsymbol{w}^{T}(s) D \boldsymbol{f}(\boldsymbol{x}(s)) \boldsymbol{w}(s) \mathrm{d} s .
\end{array}
$$

Optionally, one can invoke Birkhoff's ergodic theorem [18] and obtain the statistical average

$$
\lambda=\int \boldsymbol{w}^{T} D \boldsymbol{f}(\boldsymbol{x}) \boldsymbol{w} \mathrm{d} \rho(\boldsymbol{x}, \boldsymbol{w}),
$$

where $\rho(x, w)$ is the joint invariant distribution measure of $x$ and $\boldsymbol{w}$. The average formulas in (1.8) and (1.9) is what we need to derive the linear response approximation formula for the Lyapunov exponent below.

The manuscript is organized as follows: in Section 2 we present the formal derivation of the linear response operator for the Lyapunov exponent, in Section 3 we show the results of numerical computational tests of the derived formula with a chaotic nonlinear test model, and in Section 4 we summarize the results of the work. Additionally, Appendix A contains technical details of derivations for Section 2, while Appendix B outlines the computational discretization of the response formula. 


\section{LINEAR RESPONSE TO SMALL CONSTANT PERTURBATION}

One can introduce different types of small perturbations in the right-hand side of (1.1), and the approach, developed below, can be adapted to an arbitrary differentiable perturbation vector field. However, for simplicity of presentation, here we consider the most basic case of a constant vector perturbation $p$ :

$$
\frac{\mathrm{d} x}{\mathrm{~d} t}=f(x)+p .
$$

While this perturbation may seem trivial, there is no easy way to tell in general how the dynamical properties of (1.1) respond to such a perturbation (e.g. complex bifurcations of the resulting flow may occur, fixed points/periodic orbits created/destroyed, etc). Even if no bifurcations occur (which is what we assume here throughout the work), the solution $x(t)$ generally changes nonlinearly under finite constant perturbations, and so should do the Lyapunov exponent in (1.8) and (1.9).

Below we develop a linear approximation of the response of the Lyapunov exponent to the constant perturbation $p$ in (2.1), under the condition that $\|p\|$ is sufficiently small. For the perturbed system in (2.1), the corresponding formula for the Lyapunov exponent reads

$$
\lambda_{p}=\int w^{T} D f(x) w d \rho_{p}(x, w),
$$

where $\rho_{p}(x, w)$ is the joint invariant distribution of $x$ and $\boldsymbol{w}$ for the perturbed system in (2.1), with a shorthand notation $\rho_{0}(x, w)=\rho(x, w)$. Here we adopt the flow notations $x(t)=\phi_{p}^{t} x, w(t)=\psi_{p, x}^{t} w$, where subscripts are dropped from the initial conditions $x_{0}$ and $w_{0}$, and denote $\phi_{0}^{t}=\phi^{t}, \psi_{0, x}^{t}=\psi_{x}^{t}$. The next step is to represent $\rho_{p}(\boldsymbol{x}, \boldsymbol{w})$ as the pushforward measure [34] of $\rho(x, w)$ :

$$
\rho_{p}(x, w)=\lim _{t \rightarrow \infty} \rho\left(\phi_{p}^{-t} x, \psi_{p, x}^{-t} w\right),
$$

which yields, for $\lambda_{p}$,

$$
\begin{aligned}
& \lambda_{p}=\lim _{t \rightarrow \infty} \int \boldsymbol{w}^{T} D \boldsymbol{f}(\boldsymbol{x}) \boldsymbol{w} \mathrm{d} \rho\left(\phi_{p}^{-t} \boldsymbol{x}, \psi_{p, x}^{-t} \boldsymbol{w}\right)= \\
= & \lim _{t \rightarrow \infty} \int\left(\psi_{p, x}^{t} \boldsymbol{w}\right)^{T} D \boldsymbol{f}\left(\phi_{p}^{t} \boldsymbol{x}\right) \psi_{p, x}^{t} \boldsymbol{w} \mathrm{d} \rho(\boldsymbol{x}, \boldsymbol{w}),
\end{aligned}
$$

where the last equality follows from the change of variables $x \rightarrow \phi_{p}^{t} x, w \rightarrow$ $\psi_{p, x}^{t} \boldsymbol{w}$. Next, we use the invariance of the measure $\rho(x, w)$ with respect to the unperturbed flows $\phi^{t} \boldsymbol{x}$ and $\psi_{x}^{t} \boldsymbol{w}$ (that is, $\rho(\boldsymbol{x}, \boldsymbol{w})=\rho\left(\phi^{-t} \boldsymbol{x}, \psi_{x}^{-t} \boldsymbol{w}\right)$ for any $t$ ) and rewrite the above relation equivalently as

$$
\lambda_{p}=\lim _{t \rightarrow \infty} \int L_{p} \mathrm{~d} \rho(\boldsymbol{x}, \boldsymbol{w}),
$$




$$
L_{p}=\left(\psi_{p, \phi^{-t} x}^{t} \psi_{x}^{-t} \boldsymbol{w}\right)^{T} D f\left(\phi_{p}^{t} \phi^{-t} \boldsymbol{x}\right) \psi_{p, \phi^{-t} x}^{t} \psi_{x}^{-t} \boldsymbol{w} .
$$

Our next step is to approximate the difference $\lambda_{p}-\lambda$ via the linear relation

$$
\lambda_{p}-\lambda \approx \lim _{t \rightarrow \infty}\left(\left.\int \frac{\partial L_{p}}{\partial p}\right|_{p=0} \mathrm{~d} \rho(\boldsymbol{x}, \boldsymbol{w})\right) \cdot \boldsymbol{p},
$$

under the assumption that the derivative exists in the infinite time limit (so-called structural stability [15, 27]). Usually, it is the case with deterministic dynamical systems with Sinai-Ruelle-Bowen invariant measures [32, 35, 39], and many random dynamical systems, including Itô diffusions [17, 23]. After some calculations (which are provided in Appendix A) we obtain the linear relation in the form of the fluctuation-response time correlation functions of the unperturbed system in (1.1):

$$
\begin{gathered}
\lambda_{p}-\lambda \approx \lim _{t \rightarrow \infty} \boldsymbol{r}(t) \cdot \boldsymbol{p}, \\
\boldsymbol{r}(t)=\int_{0}^{t} \boldsymbol{c}_{1}(\tau) \mathrm{d} \tau+\int_{0}^{t} \mathrm{~d} \tau \int_{\tau}^{t} \boldsymbol{c}_{2}(\tau, s) \mathrm{d} s, \\
\boldsymbol{c}_{1}(\tau)=\lim _{T \rightarrow \infty} \frac{1}{T} \int_{0}^{T} \boldsymbol{w}\left(t^{\prime}\right)^{T} D^{2} \boldsymbol{f}\left(\boldsymbol{x}\left(t^{\prime}\right)\right): \\
:\left(\boldsymbol{w}\left(t^{\prime}\right) \otimes \boldsymbol{T}_{x\left(t^{\prime}-\tau\right)}^{\tau}\right) \mathrm{d} t^{\prime}, \\
\boldsymbol{c}_{2}(\tau, s)=\lim _{T \rightarrow \infty} \frac{1}{T} \int_{0}^{T} \boldsymbol{w}^{T}\left(t^{\prime}\right)\left(D \boldsymbol{f}\left(\boldsymbol{x}\left(t^{\prime}\right)\right)^{T}+\right. \\
\left.+D \boldsymbol{f}\left(\boldsymbol{x}\left(t^{\prime}\right)\right)\right)\left(\boldsymbol{I}-\boldsymbol{w}\left(t^{\prime}\right) \boldsymbol{w}^{T}\left(t^{\prime}\right)\right) \boldsymbol{T}_{x\left(t^{\prime}-\tau\right)}^{\tau} \times \\
\times D^{2} \boldsymbol{f}\left(\boldsymbol{x}\left(t^{\prime}-\tau\right)\right):\left(\boldsymbol{T}_{x\left(t^{\prime}\right)}^{-\tau} \boldsymbol{w}\left(t^{\prime}\right) \otimes \boldsymbol{T}_{x\left(t^{\prime}-s\right)}^{s-\tau}\right) \mathrm{d} t^{\prime} .
\end{gathered}
$$

Above, the Frobenius product ":" is computed over the two covariant indices of $D^{2} f$. The $N \times N$ matrix $\boldsymbol{T}_{x}^{t}$ is the tangent map of $\phi^{t} x$ :

$$
T_{x}^{t}=\frac{\partial}{\partial x} \phi^{t} x .
$$

The equation, which is used for the computation of the tangent map, is given in (A.6).

\section{Computational approximation AND NUMERicAl TESTING}

From the formula in (2.7) it follows that the linear approximation of the response of the Lyapunov exponent is the integral over a specially crafted time-lag correlation function with the infinite upper limit of integration. In general, the computation of such limit is feasible, as long as the integrand (the time-lag correlation under the integral) decays sufficiently rapidly to zero with increasing time, so that the integral over it could be truncated to some finite upper limit. However, 


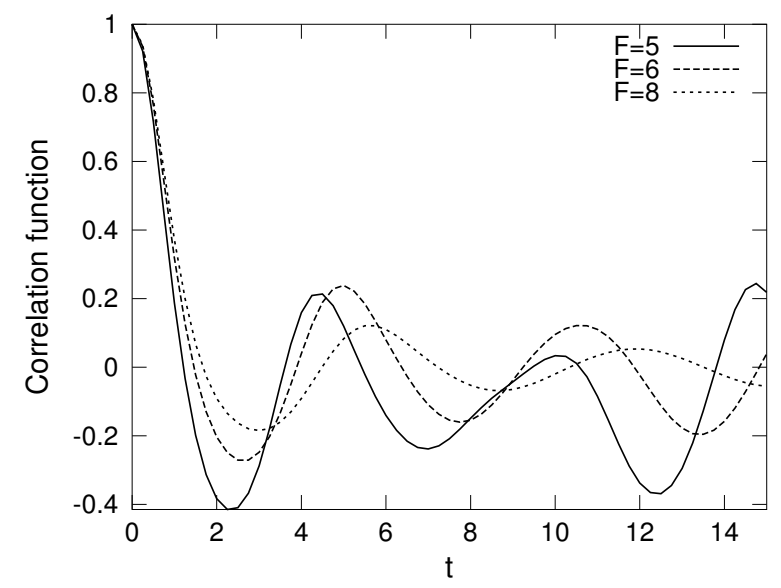

FiguRE 1. Time-lag autocorrelation functions of the 20-variable Lorenz 96 model for the regimes $F=5,6,8$.

for a chaotic and mixing dynamical system in (1.1), the situation is complicated by the fact that the tangent map $T_{x}^{t}$ of the unperturbed system in 1.11 grows exponentially rapidly in $t$ for almost any $x$, with the exponential rate of $\lambda$, even though its ensemble average decays in $t$ [19,34]. This causes the numerical instability in the form of precision loss; many extermely large numbers must add up to small numbers, which does not happen in the finite precision computer arithmetic [1-3, 12-14].

As a result, we are forced to consider a finite-time response approximation

$$
\Delta \lambda \approx \boldsymbol{r}\left(t_{0}\right) \cdot \boldsymbol{p},
$$

for a finite response time $t_{0}$, rather than an infinite time limit. The range of $t$, for which the response operator $r(t)$ is practically computable without significant numerical instability, is usually proportional to the e-folding time $\lambda^{-1}[12-14]$. The finite response time $t_{0}$ for (3.1) will have to be chosen from that range. As the test system, we consider the rescaled Lorenz 96 model [1-7, 12-14, 25, 26, 28], which is a simple nonlinear chaotic forced-dissipative system with a band of linearly unstable waves with oppositely directed phase and group velocities, similar to the Rossby waves in the midlatitudinal troposphere. The rescaled Lorenz 96 model is given by the system of ordinary differential equations

$$
\frac{\mathrm{d} x_{i}}{\mathrm{~d} t}=\left(x_{i-1}+\alpha \beta\right)\left(x_{i+1}-x_{i-2}\right)-\beta x_{i}+\beta^{2}(F-\alpha),
$$

with periodic boundary conditions $x_{0}=x_{N}$, with the total number of variables $N=20$. The parameter $F>0$ provides constant forcing, and the constant scaling parameters $\alpha>0$ and $\beta>0$ are chosen so that, for given $F$, the statistical mean state $\left\langle x_{i}\right\rangle=0$, and the statistical variance $\left\langle x_{i}^{2}\right\rangle=1$ (for details, see [28]). For 

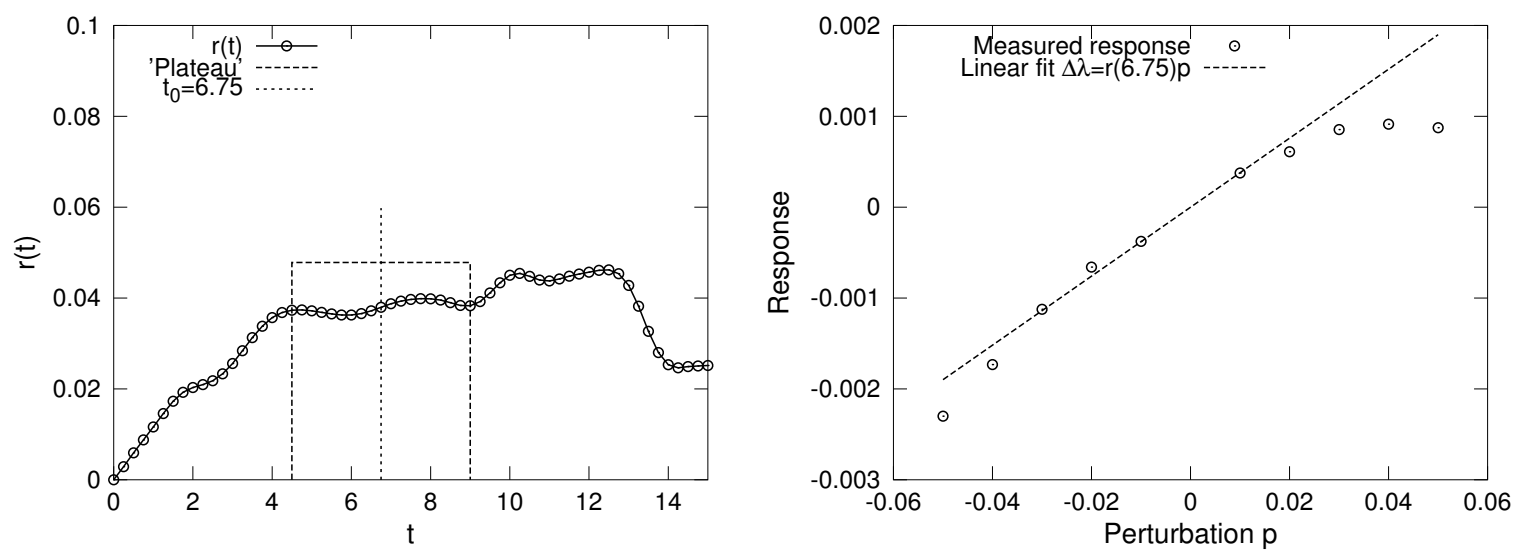

FiguRE 2. Linear response of the Lyapunov exponent for the 20variable Lorenz 96 model (3.2) with $F=5(\lambda=0.2265)$. Left: the plot $r(t)$ as a function of finite response time, and the "plateau" of saturated response before manifestation of numerical instability. The time $t_{0}=6.75$ is chosen arbitrarily from within the "plateau" window. Right: the measured response of the Lyapunov exponent (via direct perturbations) vs the linear fit $r\left(t_{0}\right) \cdot \boldsymbol{p}$.

computation of the correlation functions $c_{1}(\tau)$ and $c_{2}(\tau, s)$ from (2.7), we integrate the Lorenz 96 model in (3.2) using the standard 4th order Runge-Kutta method, with the time discretization step $\Delta t=0.01$ and the finite time averaging window $T=10^{6}$ time units. The details of numerical discretization of (2.7) are given in Appendix B. For the corresponding perturbed system, a small constant forcing $p$ is added at a single node $x_{i}$; due to the statistical translational invariance of (3.2), the number $i$ of the node is irrelevant. For the same reason, the entries of $r(t)$ are identical, $r_{i}(t)=r(t)$. Thus, the linear response approximation of the Lyapunov exponent here is given by

$$
\Delta \lambda \approx r\left(t_{0}\right) p .
$$

For the test, we pick three different values of $F=5,6$ and 8 , which correspond to the dynamical regimes of low, moderate and strong chaos and mixing, respectively. The standard time-lagged autocorrelation functions $\left\langle x_{i}(t) x_{i}(t+\tau)\right\rangle$ (which are also identical across different $i$ due to translational invariance of (3.2)) for these regimes are shown in Figure 1 as functions of the time lag. Observe that the initial time scales of decorrelation are identical (the correlation functions are almost the same for short lags), which is the effect of rescaling by parameters $\alpha$ and $\beta$ in (3.2). For longer correlation lags we, however, can observe better mixing (more rapid decay of lag-correlations) for the regimes $F=6,8$. The corresponding values of the Lyapunov exponent for these regimes are $\lambda=0.2265,0.3024$ and 0.4253 , respectively. In Figures $2-4$ we demonstrate the computed finite-time 

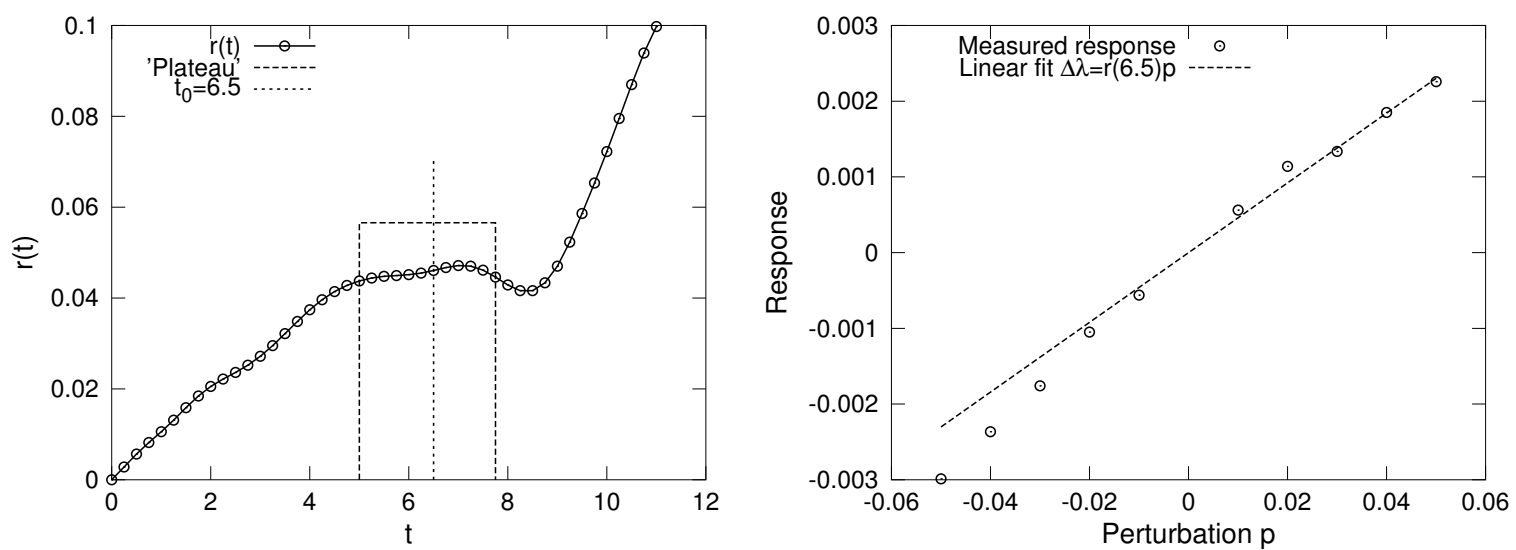

FiguRE 3. Linear response of the Lyapunov exponent for the 20variable Lorenz 96 model (3.2) with $F=6(\lambda=0.3024)$. Left: the plot $\boldsymbol{r}(t)$ as a function of finite response time, and the "plateau" of saturated response before manifestation of numerical instability. The time $t_{0}=6.5$ is chosen arbitrarily from within the "plateau" window. Right: the measured response of the Lyapunov exponent (via direct perturbations) vs the linear fit $\boldsymbol{r}\left(t_{0}\right) \cdot \boldsymbol{p}$.

response operators $r(t)$ for the regimes of the Lorenz 96 model with $F=5,6,8$, as well as the actual directly perturbed and measured responses of the Lyapunov exponents for the perturbed system and corresponding finite-time linear response approximations. We observe that the temporal behavior of $r(t)$ generally consists of three stages:

(1) The initial growth stage, since $r(t)$ always starts at zero response for $t=0$.

(2) The "plateau" stage, where the response has grown close to its equilibrium value. This stage should be the best approximation to the actual response of the Lyapunov exponent.

(3) The blow-up stage, where the numerical instability in $T_{x}^{t}$ manifests itself. This stage is characterized by irregular oscillations and further growth of $r(t)$.

The "plateau" stage of the linear response $r(t)$ is identified on each plot, and the times $t_{0}$ for the finite-time response approximations are chosen from within the plateau stages for corresponding plots. It is not known at present whether the Lorenz 96 model is structurally stable in the displayed regimes (most likely not), yet, structural instability does not appear to manifest itself in the directly perturbed response significantly (that is, the circles in the right-hand plots in Figures 2 -4 appear to lie on a "smooth" curve, rather than on some kind of a discontinuous set). Observe that the actual response of the Lyapunov exponent to the perturbation is generally nonlinear, however, there is a range of linearity around 

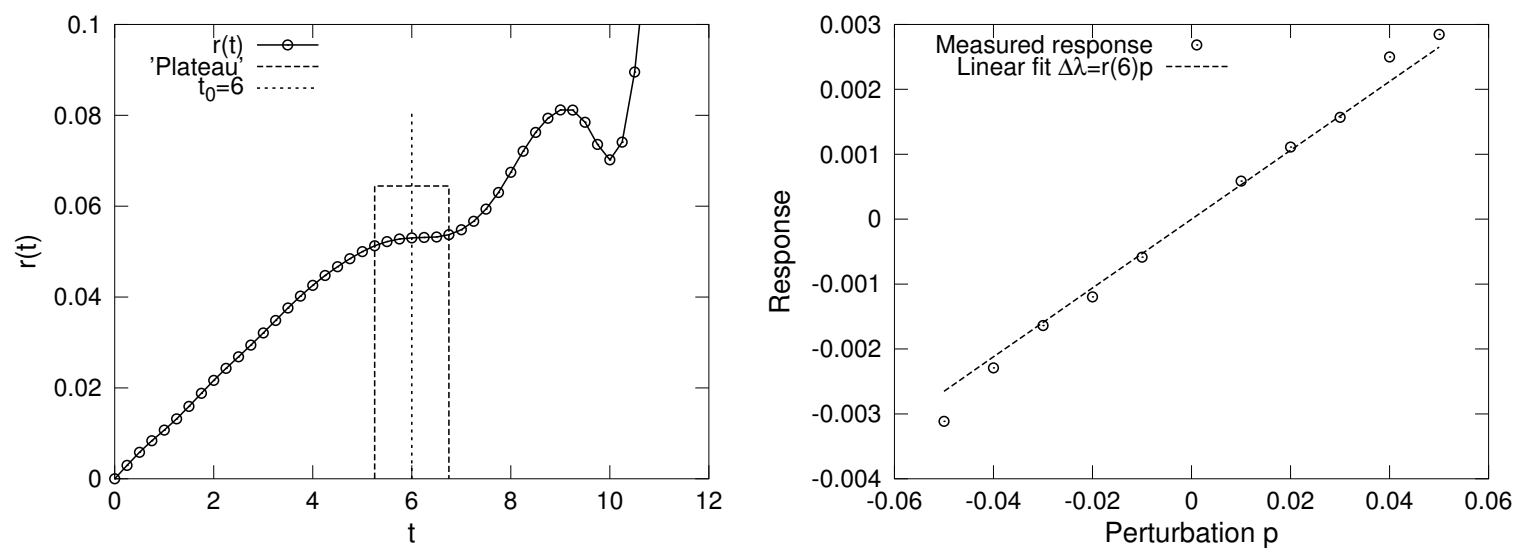

FiguRE 4. Linear response of the Lyapunov exponent for the 20variable Lorenz 96 model (3.2) with $F=8(\lambda=0.4253)$. Left: the plot $r(t)$ as a function of finite response time, and the "plateau" of saturated response before manifestation of numerical instability. The time $t_{0}=6$ is chosen arbitrarily from within the "plateau" window. Right: the measured response of the Lyapunov exponent (via direct perturbations) vs the linear fit $r\left(t_{0}\right) \cdot \boldsymbol{p}$.

the unperturbed state, which appears to depend on the dynamical regime. It is the most narrow in the weakly chaotic regime $(F=5)$, where the smallest perturbations used, $p= \pm 0.01$ yield linear response, however, larger perturbations ( $p= \pm 0.02$ and above) cause a distinctly nonlinear, "parabolic" shape of the response plot. As the dynamical regime becomes more chaotic and mixing, the range of linearity becomes extended ( $p= \pm 0.02$ for $F=6$, and $p= \pm 0.03$ for $F=8$ ). In the range of linearity, the finite-time linear response approximation yields a good fit to the perturbed response of the Lyapunov exponent for each dynamical regime, although an "undershot" (an insufficiently steep slope) can be seen for the moderately chaotic and mixing dynamical regime $F=6$. Thus, it appears that the weak chaos in the dynamics sets the natural bound of applicability of the linearized formula, due to inherent nonlinearity of the perturbed response of the Lyapunov exponent. The additional observed effect is that the "plateau" of the response approximation formula (2.7) becomes more narrow with the increase in chaos, due to the fact that the numerical instability in the exponentially growing tangent map manifests itself earlier in time. This naturally leads to the speculation that, for strongly turbulent dynamical regimes, the numerical instability could occur even before the initial response growth stage is completed, thus leading to the absence of a discernible "plateau", thus setting another bound of practical applicability of the linear response formula in (2.7). Overall, for the regimes considered, the key "plateau" stages in each response operator $r(t)$ are clearly identifiable by sight in each plot, and the linear response fits, provided by 
the finite time linear approximation from within these "plateau" stages, seem to be adequate approximations to the directly perturbed responses of the Lyapunov exponent.

\section{Conclusions}

In the current work we develop a linear approximation to the response of the Lyapunov exponent of a nonlinear chaotic dynamical system to a small constant perturbation. This approximation is computed from a long-term trajectory of the corresponding unperturbed system. The approximation is based on the fluctuation-dissipation theorem approach [1-3, 12, 14, 34] using the tangent map of the underlying chaotic dynamical system. We numerically test the new approach using the rescaled Lorenz 96 model [1-7, 12, 14, 25, 26, 28] with 20 variables, in three dynamical regimes: weakly, moderately, and strongly chaotic. We show that, despite the fact that an inherent numerical instability due to exponentially growing in time tangent map renders the formal infinite time limit infeasible for practical computation, a finite-time linear response formula adequately approximates the actual perturbed values of the Lyapunov exponent in their range of linearity, for the regimes considered. We also observe that, for the same range of perturbations, the nonlinearity of the perturbed response is strongest in the weakly chaotic regime, and, vice-versa, weakest in the strongly chaotic regime. Also, the "plateau" stage of the linear response approximation between the initial growth and numerical instability, which is crucial for choosing the correct finite response time, appears to shrink when the dynamical regime is strongly chaotic. It leads to the speculation that for strongly turbulent regimes the developed method could be rendered inapplicable due to the complete absence of the "plateau" stage. Thus, the range of practical applicability of the method appears to be limited by the response nonlinearity on the weak chaos side, and by the rapidly developing numerical instability in the tangent map on the strong chaos side.

In the future work, we plan to investigate the response of the Lyapunov exponent to linear vector field perturbations. Under special interest are conservative systems whose solutions preserve a quadratic energy (such as the truncated Burgers-Hopf system, the Kruskal-Zabusky system, and unforced, undamped Lorenz 96 system [8-11]). In such systems, a skew-symmetric (in the energy metric) linear perturbation vector field will preserve the solutions on the same constant energy surface, at the same time affecting chaos and turbulence of the system. An interesting problem would be the maximization of the Lyapunov exponent under linear skew-symmetric perturbation for given nonlinear conservative dynamics, constrained to a fixed constant energy surface.

Acknowledgments. This work was supported by the National Science Foundation CAREER grant DMS-0845760. 


\section{Appendix A. Details on Derivation}

The chain rule, applied to $L_{p}$ in (2.6), yields

$$
\begin{gathered}
\left.\frac{\partial L_{p}}{\partial p}\right|_{p=0}=\left.\left.\frac{\partial L_{p}}{\partial \phi_{p}^{t} \phi^{-t} \boldsymbol{x}}\right|_{\phi_{p}^{t} \phi^{-t} x=x} \frac{\partial \phi_{p}^{t} \phi^{-t} x}{\partial p}\right|_{p=0}+ \\
+\left.\left.\frac{\partial L_{p}}{\partial \psi_{p, \phi^{-t} x}^{t} \psi_{x}^{-t} \boldsymbol{w}}\right|_{\psi_{p, \phi^{-} t_{x}}^{t} \psi_{x}^{-t} w=w} \frac{\partial \psi_{p, \phi^{-t} x}^{t} \psi_{x}^{-t} \boldsymbol{w}}{\partial p}\right|_{p=0},
\end{gathered}
$$

where the corresponding partial derivatives are given by

$$
\left.\frac{\partial L_{p}}{\partial \phi_{p}^{t} \phi^{-t} x}\right|_{\phi_{p}^{t} \phi^{-t} x=x}=\boldsymbol{w}^{T} D^{2} f(x) w,
$$

$$
\left.\frac{\partial L_{p}}{\partial \psi_{p, \phi^{-t} x}^{t} \psi_{x}^{-t} \boldsymbol{w}}\right|_{\psi_{p, \phi^{-t_{x}}}^{t} \psi_{x}^{-t} w=w}=\left(D \boldsymbol{f}(\boldsymbol{x})+D \boldsymbol{f}(\boldsymbol{x})^{T}\right) \boldsymbol{w} .
$$

Now we denote $y=\phi^{-t} x$ and compute $\partial \phi_{p}^{t} y /\left.\partial p\right|_{p=0}$. Observe that $\phi_{p}^{t} y$ satisfies

$$
\frac{\partial}{\partial t} \phi_{p}^{t} y=f\left(\phi_{p}^{t} y\right)+p,
$$

where the differentiation on both sides with respect to $p$ yields the linear equation

$$
\frac{\partial}{\partial t}\left(\frac{\partial \phi_{p}^{t} y}{\partial p}\right)=D f\left(\phi_{p}^{t} y\right) \frac{\partial \phi_{p}^{t} y}{\partial p}+I,
$$

where $\boldsymbol{I}$ is the identity matrix. Upon replacing $y$ back with $\phi^{-t} \boldsymbol{x}$, the solution is given

$$
\left.\frac{\partial \phi_{p}^{t} \phi^{-t} x}{\partial p}\right|_{p=0}=\int_{0}^{t} \boldsymbol{T}_{\phi^{-\tau} x}^{\tau} \mathrm{d} \tau,
$$

where the tangent map $T_{p, x}^{t}=\partial \phi_{p}^{t} x / \partial x$ is computed from the equation

$$
\frac{\partial}{\partial t} T_{p, x}^{t}=D f\left(\phi_{p}^{t} x\right) T_{p, x}^{t}, \quad T_{p, x}^{0}=I .
$$

For $\partial\left(\psi_{p, \phi^{-} x}^{t} \psi_{x}^{-t} \boldsymbol{w}\right) /\left.\partial p\right|_{p=0}$, we again denote $\boldsymbol{y}=\phi^{-t} \boldsymbol{x}$, and, additionally, $\boldsymbol{z}=$ $\psi_{x}^{-t} w$, thus switching to the computation of $\partial\left(\psi_{p, y}^{t} z\right) /\left.\partial g\right|_{p=0}$. At this point, recall 
that $w=v /\|v\|$, where the tangent vector is given by $v=T_{p, y}^{t} z$. Using the chain rule of differentiation again, we obtain

$$
\left.\frac{\partial \psi_{p, y}^{t} z}{\partial p}\right|_{p=0}=\left.\frac{\partial w}{\partial v} \frac{\partial T_{p, y}^{t} z}{\partial p}\right|_{p=0}
$$

The term $\partial T_{p, y}^{t} z / \partial p$ satisfies (A.6), differentiated by $p$ on both sides:

$$
\begin{aligned}
& \frac{\partial}{\partial t}\left(\frac{\partial T_{p, y}^{t} z}{\partial p}\right)=D f\left(\phi_{p}^{t} y\right) \frac{\partial T_{p, y}^{t} z}{\partial p}+ \\
& \quad+D^{2} f\left(\phi_{p}^{t} y\right):\left(T_{p, y}^{t} z \otimes \frac{\partial \phi_{p}^{t} y}{\partial p}\right)
\end{aligned}
$$

where the Frobenius product ":" is computed over the two covariant indices of $D^{2} f$. The solution is given by

$$
\begin{array}{r}
\left.\frac{\partial T_{p, x}^{t} z}{\partial p}\right|_{p=0}=\int_{0}^{t} T_{\phi^{\tau} y}^{t-\tau} D^{2} f\left(\phi^{\tau} y\right): \\
:\left(T_{y}^{\tau} z \otimes \int_{0}^{\tau} T_{\phi^{s} y}^{\tau-s} \mathrm{~d} s\right) \mathrm{d} \tau,
\end{array}
$$

where we took into account (A.5). Now, observe that

$$
T_{y}^{\tau} z=\|v\| T_{\phi^{-t} x}^{\tau} T_{x}^{-t} w=\|v\| T_{x}^{\tau-t} w
$$

which, upon substitution into A.9 and rearrangement of dummy variables of integration, yields

$$
\begin{aligned}
\left.\frac{\partial T_{p, x}^{t} z}{\partial p}\right|_{p=0} & =\|v\| \int_{0}^{t} T_{\phi^{-\tau} x}^{\tau} D^{2} f\left(\phi^{-\tau} x\right): \\
& :\left(T_{x}^{-\tau} w \otimes \int_{\tau}^{t} T_{\phi^{-s} x}^{s-\tau} \mathrm{d} s\right) \mathrm{d} \tau .
\end{aligned}
$$

Next we compute

$$
\frac{\partial w}{\partial v}=\frac{1}{\|v\|}\left(I-w w^{T}\right)
$$


which, together with (A.11, yields

$$
\begin{gathered}
\left.\frac{\partial \psi_{p, \phi^{-t_{x}}}^{t} \psi_{x}^{-t} \boldsymbol{w}}{\partial \boldsymbol{p}}\right|_{p=0}=\left(\boldsymbol{I}-\boldsymbol{w} \boldsymbol{w}^{T}\right) \int_{0}^{t} \boldsymbol{T}_{\phi^{-\tau} \boldsymbol{x}}^{\tau} \times \\
\times D^{2} \boldsymbol{f}\left(\phi^{-\tau} \boldsymbol{x}\right):\left(\boldsymbol{T}_{x}^{-\tau} \boldsymbol{w} \otimes \int_{\tau}^{t} \boldsymbol{T}_{\phi^{-s} x}^{s-\tau} \mathrm{d} s\right) \mathrm{d} \tau .
\end{gathered}
$$

Combining the computed terms together under (2.6), we obtain

$$
\begin{gathered}
\lambda^{*}-\lambda \approx \lim _{t \rightarrow \infty} \boldsymbol{r}(t) \cdot \boldsymbol{p}, \\
\boldsymbol{r}(t)=\int_{0}^{t} c_{1}(\tau) \mathrm{d} \tau+\int_{0}^{t} \mathrm{~d} \tau \int_{\tau}^{t} c_{2}(\tau, s) \mathrm{d} s, \\
c_{1}(\tau)=\int \boldsymbol{w}^{T} D^{2} \boldsymbol{f}(\boldsymbol{x}):\left(\boldsymbol{w} \otimes \boldsymbol{T}_{\phi^{-\tau} \boldsymbol{x}}^{\tau}\right) \mathrm{d} \rho(\boldsymbol{x}, \boldsymbol{w}), \\
\boldsymbol{c}_{2}(\tau, s)=\int \boldsymbol{w}^{T}\left(D \boldsymbol{f}(\boldsymbol{x})+D \boldsymbol{f}(\boldsymbol{x})^{T}\right) \times \\
\times\left(\boldsymbol{I}-\boldsymbol{w} \boldsymbol{w}^{T}\right) \boldsymbol{T}_{\phi^{-\tau} \boldsymbol{x}}^{\tau} D^{2} \boldsymbol{f}\left(\phi^{-\tau} \boldsymbol{x}\right): \\
:\left(\boldsymbol{T}_{\boldsymbol{x}}^{-\tau} \boldsymbol{w} \otimes \boldsymbol{T}_{\phi^{-s} \boldsymbol{x}}^{s-\tau}\right) \mathrm{d} \rho(\boldsymbol{x}, \boldsymbol{w}) .
\end{gathered}
$$

Invoking Birkhoff's ergodic theorem and replacing the measure averages with long-term time averages, we obtain the correlation functions in (2.7).

\section{Appendix B. Details on Discretization}

We discretize $c_{1}(\tau)$ and $c_{2}(\tau, s)$ from (2.7) as follows: first, we assume that $\tau$ can assume a finite range of values $\tau_{m}=\{0 \ldots h m \ldots h M\}$, where $h$ is the discretization step, and $M$ is a positive integer, with $h M$ bounding the range of possible values of $\tau$ from above. For each value $h m$ of $\tau, s$ assumes the values $s_{n}=$ $\{h m \ldots h n \ldots h M\}$, so that the array of discretized values $\left(\tau_{m}, s_{n}\right)$ is triangular. Then, the integrals over $c_{1}\left(\tau_{m}\right)$ and $c_{2}\left(\tau_{m}, s_{n}\right)$ in (2.7) are computed using the standard trapezoidal quadrature rule.

The discretized correlation functions $c_{1}\left(\tau_{m}\right), c_{2}\left(\tau_{m}, s_{n}\right)$ are computed as follows. Let us first discretize the trajectory $x(t), w(t)$ into the set of vectors $x_{k}=x\left(t_{k}\right)$, $w_{k}=w\left(t_{k}\right)$, where $k$ is the discretization index of time $t$. Let us also denote the incremental (forward by $h$ ) tangent map $\boldsymbol{T}_{x\left(t_{k}\right)}^{h}=\boldsymbol{T}_{k}$. Then, any tangent map $T_{x\left(t_{k}\right)}^{h i}$ can be written as a product of the incremental tangent maps $[1-3]$

$$
\boldsymbol{T}_{\boldsymbol{x}\left(t_{k}\right)}^{h i}=\prod_{j=0}^{i-1} \boldsymbol{T}_{k+j}
$$


Additionally, the backward (in time) tangent maps are readily available as inverses of the corresponding forward tangent maps:

$$
T_{k}^{-h}=\left(T_{k-1}^{h}\right)^{-1}
$$

The incremental tangent maps $\boldsymbol{T}_{k}$ are obtained naturally by solving the linear equation (A.6) with $p=0$ along the trajectory $x(t)$ between $t_{k}$ and $t_{k}+h$. Then, the time-averaged formulas for $c_{1}(\tau)$ and $c_{2}(\tau)$ in (2.7) are expressed as the following discretized averages:

$$
\begin{gathered}
\boldsymbol{c}_{1}\left(\tau_{m}\right)=\lim _{K \rightarrow \infty} \frac{1}{K} \sum_{k=M}^{K+M-1} \boldsymbol{w}_{k}^{T} D^{2} \boldsymbol{f}\left(\boldsymbol{x}_{k}\right): \\
:\left(\boldsymbol{w}_{k} \otimes \prod_{j=0}^{m-1} \boldsymbol{T}_{k-m+j}\right), \quad 0 \leq m \leq M, \\
\boldsymbol{c}_{2}\left(\tau_{m}, s_{n}\right)=\lim _{K \rightarrow \infty} \frac{1}{K} \sum_{k=M}^{K+M-1} \boldsymbol{w}_{k}^{T}\left(D \boldsymbol{f}\left(\boldsymbol{x}_{k}\right)^{T}+\right. \\
\left.+D \boldsymbol{f}\left(\boldsymbol{x}_{k}\right)\right)\left(\boldsymbol{I}-\boldsymbol{w}_{k} \boldsymbol{w}_{k}^{T}\right) \prod_{j=0}^{m-1} \boldsymbol{T}_{k-m+j} D^{2} \boldsymbol{f}\left(\boldsymbol{x}_{k-m}\right): \\
:\left(\prod_{j=0}^{m-1}\left(\boldsymbol{T}_{k-j}\right)^{-1} \boldsymbol{w}_{k} \otimes \prod_{j=0}^{n-m} \boldsymbol{T}_{k-n+j}\right), \quad m \leq n \leq M .
\end{gathered}
$$

The formulas above are computed "on-the-fly" along with a discretized long-term trajectory $\boldsymbol{x}_{k}, \boldsymbol{w}_{k}, \boldsymbol{T}_{k}$, with the only memory requirement is that, as the discretized trajectory is computed, the last $M$ incremental tangent maps $\boldsymbol{T}_{k}$ are temporarily stored for computation, where $M$ is a fixed number. Note that $h$ above is not necessarily the discretization time step for the 4th order Runge-Kutta integrator of the unperturbed equation in (1.1); in particular, here we use $h=0.25$ time units (with $M=60$, so that the response time spanned by $r(t)$ is at most 15 time units), while the Runge-Kutta time step is $\Delta t=0.01$ (that is, the averages in (B.3) are updated once per 25 Runge-Kutta steps). This allows to balance the workload in a multithreaded implementation of the algorithm, where the computation of $\boldsymbol{x}_{k}$, $w_{k}, T_{k}$, and the updates to (B.3) are spread between different CPUs. The time averaging window is $T=10^{6}$ time units, which sets $K=4 \cdot 10^{6}$.

\section{REFERENCES}

[1] R.V. Abramov. Short-time linear response with reduced-rank tangent map. Chin. Ann. Math., 30B(5):447-462, 2009.

[2] R.V. Abramov. Approximate linear response for slow variables of deterministic or stochastic dynamics with time scale separation. J. Comput. Phys., 229(20):7739-7746, 2010. 
[3] R.V. Abramov. Improved linear response for stochastically driven systems. Front. Math. China, 7(2):199-216, 2012.

[4] R.V. Abramov. A simple linear response closure approximation for slow dynamics of a multiscale system with linear coupling. Multiscale Model. Simul., 10(1):28-47, 2012.

[5] R.V. Abramov. Suppression of chaos at slow variables by rapidly mixing fast dynamics through linear energy-preserving coupling. Commun. Math. Sci., 10(2):595-624, 2012.

[6] R.V. Abramov. A simple closure approximation for slow dynamics of a multiscale system: Nonlinear and multiplicative coupling. Multiscale Model. Simul., 11(1):134-151, 2013.

[7] R.V. Abramov. A simple stochastic parameterization for reduced models of multiscale dynamics. J. Comput. Dynamics, 2013. submitted.

[8] R.V. Abramov, G. Kovačič, and A.J. Majda. Hamiltonian structure and statistically relevant conserved quantities for the truncated Burgers-Hopf equation. Comm. Pure Appl. Math., 56:0001-0046, 2003.

[9] R.V. Abramov and A.J. Majda. Discrete approximations with additional conserved quantities: Deterministic and statistical behavior. Meth. Appl. Anal., 10(2):151-190, 2003.

[10] R.V. Abramov and A.J. Majda. Quantifying uncertainty for non-Gaussian ensembles in complex systems. SIAM J. Sci. Comp., 26(2):411-447, 2003.

[11] R.V. Abramov and A.J. Majda. Statistically relevant conserved quantities for truncated quasigeostrophic flow. Proc. Natl. Acad. Sci., 100(7):3841-3846, 2003.

[12] R.V. Abramov and A.J. Majda. Blended response algorithms for linear fluctuation-dissipation for complex nonlinear dynamical systems. Nonlinearity, 20:2793-2821, 2007.

[13] R.V. Abramov and A.J. Majda. New approximations and tests of linear fluctuation-response for chaotic nonlinear forced-dissipative dynamical systems. J. Nonlin. Sci., 18(3):303-341, 2008.

[14] R.V. Abramov and A.J. Majda. New algorithms for low frequency climate response. J. Atmos. Sci., 66:286-309, 2009.

[15] D. Albers and J. Sprott. Structural stability and hyperbolicity violation in high-dimensional dynamical systems. Nonlinearity, 19:1801-1847, 2006.

[16] H. Aref. Stirring by chaotic advection. J. Fluid Mech., 143:1-21, 1984.

[17] L. Arnold. Random Dynamical Systems. Springer Monographs in Mathematics. Springer, 2002.

[18] G.D. Birkhoff. Proof of the ergodic theorem. Proc. Natl. Acad. Sci., 17(12):656-660, 1931.

[19] J.-P. Eckmann and D. Ruelle. Ergodic theory of chaos and strange attractors. Rev. Mod. Phys., 57(3):617-656, 1985.

[20] E. Kalnay. Atmospheric Modeling, Data Assimilation and Predictability. Cambridge University Press, New York, 2003.

[21] H. Kantz. A robust method to estimate the maximal Lyapunov exponent of a time series. Phys. Lett. A, 185(1):77-87, 1985.

[22] R. Kraichnan. An almost-Markovian Galilean-invariant turbulence model. J. Fluid Mech., 47:513-524, 1971.

[23] H. Kunita. Stochastic flows and stochastic differential equations. Cambridge University Press, 1997.

[24] E. Lorenz. Deterministic non-periodic flow. J. Atmos. Sci., 20:130-141, 1963.

[25] E. Lorenz. Predictability: A problem partly solved. In Proceedings of the Seminar on Predictability, Shinfield Park, Reading, England, 1996. ECMWF.

[26] E. Lorenz and K. Emanuel. Optimal sites for supplementary weather observations. J. Atmos. Sci., 55:399-414, 1998.

[27] R. Mañe. A proof of the $C^{1}$ stability conjecture. Publ. Math. IHES, 66:161-210, 1988. 
[28] A.J. Majda, R.V. Abramov, and M.J. Grote. Information Theory and Stochastics for Multiscale Nonlinear Systems, volume 25 of CRM Monograph Series of Centre de Recherches Mathématiques, Université de Montréal. American Mathematical Society, 2005. ISBN 0-8218-3843-1.

[29] V. Oseledec. A multiplicative ergodic theorem: Lyapunov characteristic numbers for dynamical systems. Trans. Mosc. Math. Soc., 19:197-231, 1968.

[30] E. Ott. Chaos in Dynamical Systems. Cambridge University Press, 1993.

[31] H. Risken. The Fokker-Planck Equation. Springer-Verlag, New York, 2nd edition, 1989.

[32] D. Ruelle. A measure associated with Axiom A attractors. Amer. J. Math., 98:619-654, 1976.

[33] D. Ruelle. Measures describing a turbulent flow. Ann. NY Acad. Sci., 357:1-9, 1980.

[34] D. Ruelle. General linear response formula in statistical mechanics, and the fluctuationdissipation theorem far from equilibrium. Phys. Lett. A, 245:220-224, 1998.

[35] Ya. Sinai. Gibbs measure in ergodic theory. Russian Math. Surveys, 27:21-69, 1972.

[36] S. Strogatz. Nonlinear Dynamics and Chaos: with Applications to Physics, Biology, Chemistry, and Engineering. Addison-Wesley Publishing Company, 1994.

[37] J. Weber. Fluctuation Dissipation theorem. Phys. Rev., 101(6):1620-1626, 1956.

[38] A. Wolf, J.B. Swift, H.L. Swinney, and J.A. Vastano. Determining Lyapunov exponents from a time series. Physica D, 16(3):285-317, 1985.

[39] L.-S. Young. What are SRB measures, and which dynamical systems have them? J. Stat. Phys., 108(5-6):733-754, 2002.

Department of Mathematics, Statistics and Computer Science, University of Illinois at Chicago, 851 S. Morgan st. (M/C 249), Chicago, IL 60607

E-mail address: abramov@math.uic.edu 